\title{
Review of: "The role of sheep ked (Melophagus ovinus) as potential vector of protozoa and bacterial pathogens"
}

\author{
Hans-Peter Fuehrer ${ }^{1}$ \\ 1 University of Veterinary Medicine, Vienna
}

Potential competing interests: The author(s) declared that no potential competing interests exist.

The authors describe the findings of DNA of various bacteria and protozoa in sheep keds in Poland. The manuscript is short and includes a low number of sheep keds $(n=129)$. Some pathogens are not specified to species level (and for certain bacteria more than one PCR is run). Overall, the manuscript provides some interesting data. However - phylogenetic trees would increase the relevance of the manuscript.

Major comment:

The findings of DNA of certain pathogens in a sheep ked or other vector does neither prove vector competence nor vector capacity. The finding of Borrelia burgdorferi is no prove for vector competence.

Title - include Poland

Introduction - blood sucking flies sounds strange - change to louse flies, dipterans or insects

Check Hippoboscidae and Hippoboscinae

The sheep ked is discussed to be a competent vector of zoonotic pathogens - vector capacity is not proven and if it is - cite 